\title{
Choriocarcinoma with multiple lung, skull and skin metastases in a postmenopausal female: A case report
}

\author{
XIAO-MAN LI, XIAO-YUN LIU and ZHI-XIA LIU \\ Department of Gynecology, The Third Affiliated Hospital of Zunyi Medical College, Zunyi, Guizhou 563000 P.R. China
}

Received October 23, 2014; Accepted August 20, 2015

DOI: $10.3892 / \mathrm{ol} .2015 .3750$

\begin{abstract}
Gestational trophoblastic disease with a primary extra-uterine nidus is rare, particularly during the postmenopausal period. The present report outlines a case of high-risk choriocarcinoma (International Federation of Gynecology and Obstetrics stage IV; World Health Organization score 13) in a 68 -year-old female exhibiting neoplasm. The choriocarcinoma developed 20 years subsequent to the onset of menopause and 42 years following the patient's final pregnancy, and was associated with multiple metastases to the lungs, skull, neck, lymph nodes and skin. The patient was administered two courses of systemic chemotherapy with tegafur $(800 \mathrm{mg}$ ) and actinomycin D $(200 \mu \mathrm{g})$. Local chemotherapy was also administered to the scalp and left flank masses; the masses were injected first with methotrexate, and then with 5-fluorouracil. During chemotherapy, the patient's levels of $\beta$-human chorionic gonadotrophin ( $\beta$-HCG) decreased from 3,171 IU/1 to $1,763 \mathrm{IU} / 1$, however by the conclusion of the courses of systemic and local chemotherapy, $\beta$-HCG levels had increased to $3,704 \mathrm{IU} / 1$. The patient and their family subsequently elected to end treatment. The patient subsequently succumbed to infection, tumor consumption and organ insufficiency. This study describes the clinical and radiological features, as well as the treatment used for this rare type of choriocarcinoma.
\end{abstract}

\section{Introduction}

Choriocarcinoma is a rare and highly malignant neoplasm classed among the gestational trophoblastic diseases (1). Choriocarcinoma is associated with molar pregnancy, a rapid rate of hematogenous spread to multiple organs and increased levels of $\beta$-human chorionic gonadotropin $(\beta-\mathrm{HCG})$, and is also associated with a positive response to

Correspondence to: Professor Xiao-Yun Liu, Department of Gynecology, The Third Affiliated Hospital of Zunyi Medical College, 98 Fenghuang Road, Zunyi, Guizhou 563000, P.R. China E-mail: 656479556@qq.com

Key words: gestational trophoblastic disease, non-gestational trophoblastic disease, choriocarcinoma, multiple metastases, postmenopausal chemotherapy $(2,3)$. Choriocarcinoma primarily occurs in females of childbearing age and is rarely observed subsequent to menopause (1). Primary symptoms outside the uterus, including lung, bone and skin metastases, are particularly uncommon. The present report outlines a case of postmenopausal high-risk choriocarcinoma [International Federation of Gynecology and Obstetrics (FIGO) stage IV, World Health Organization (WHO) score 13] in a 68-year-old female, who was identified due to the presence of a pulmonary lesion and surface masses. Written informed consent was obtained from the patient's family.

\section{Case report}

A 68-year-old female, presenting with a prolonged cough with expectoration for three months, was admitted to the Pneumology Department of The Third Affiliated Hospital of Zunyi Medical College (Zunyi, China). A chest computed tomography (CT) scan, performed three months prior to admission, had revealed a number of nodular opacities with blurred edges in the right upper lobe of the lung. The largest opacity measured $3.6 \times 3.7 \mathrm{~cm}$, and exhibited focal cavitation, without enlargement of intrapulmonary or mediastinal lymph nodes. A diagnosis of a lung abscess due to secondary pulmonary tuberculosis was reached, and the patient was administered cefotaxime ( $2 \mathrm{~g}$, every $12 \mathrm{~h}$ ) and levofloxacin ( $0.2 \mathrm{~g}$, every $12 \mathrm{~h})$ for 2 weeks to treat the infection, and rifampin $(0.45 \mathrm{~g}$, once daily), isoniazid ( $0.3 \mathrm{~g}$, once daily) and ethambutol $(0.75 \mathrm{~g}$, once daily) for 3 months to treat the tuberculosis. However, the patient was subsequently readmitted to hospital three months later, exhibiting aggravated symptoms, including headaches and anorexia. Once admitted, the patient continued to receive the therapy regime comprised of the aforementioned antibiotics and anti-tuberculosis medication. On day 6 following admission, the patient became febrile $\left(38-39.7^{\circ} \mathrm{C}\right)$. Lymph nodes of the neck were swollen, and two masses were identified on the patient's scalp, measuring $\sim 3 x 3$ and $3 \times 4 \mathrm{~cm}$, respectively. Consequently, a lymph node biopsy was performed, during which cytological examination detected the presence of oncocytes. Serum tumor markers were within normal ranges, with the exception of $\beta$-HCG and carbohydrate antigen 125 , which were elevated (3,171 IU/1 and $96.7 \mathrm{U} / \mathrm{ml}$, respectively). At this point, the patient was transferred to the Department of Gynecology for the continuation of treatment, due to the consideration of a diagnosis of choriocarcinoma. Speculum 


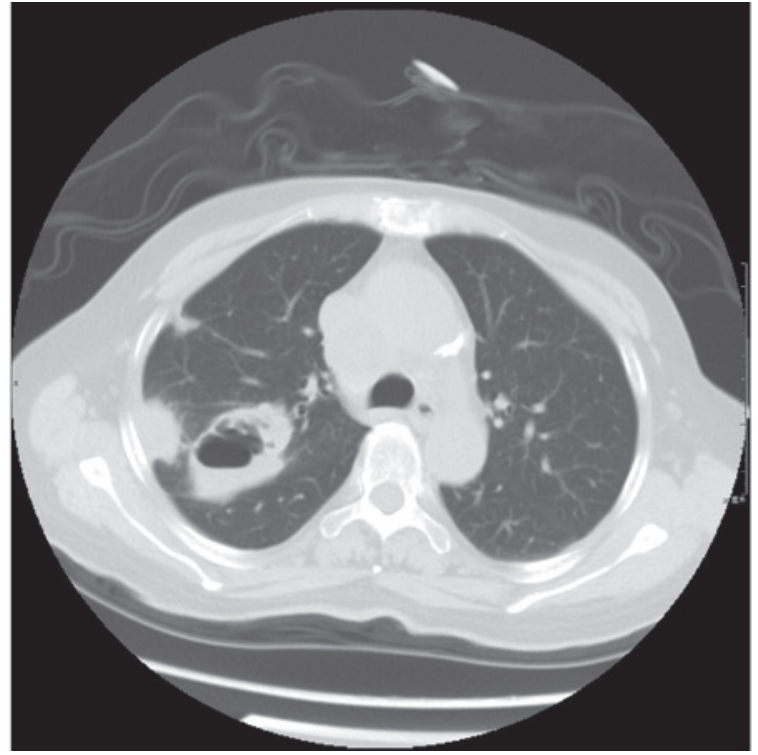

Figure 1. Computed tomography scan of the pulmonary lesion.

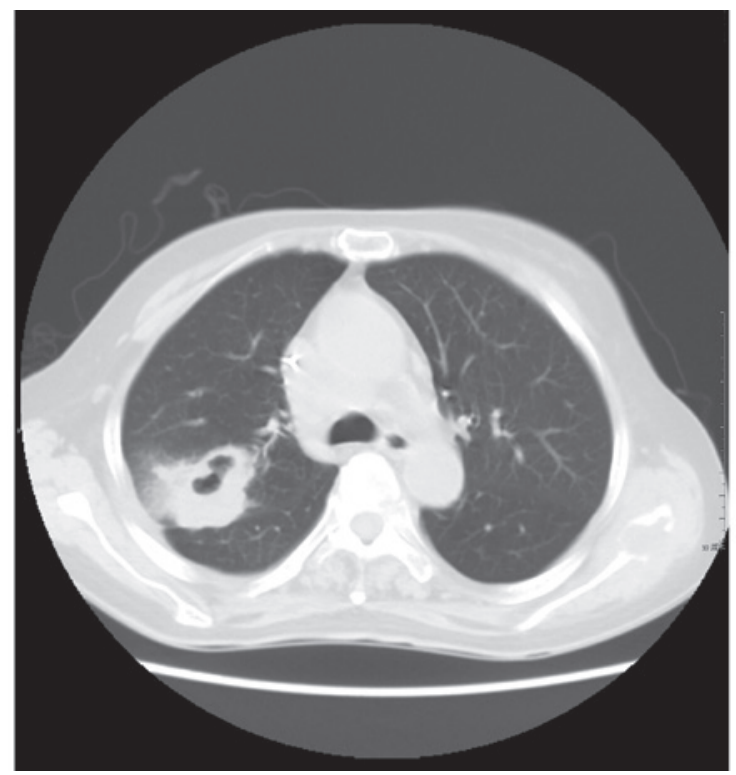

Figure 2. Computed tomography scan of the scapula metastases.

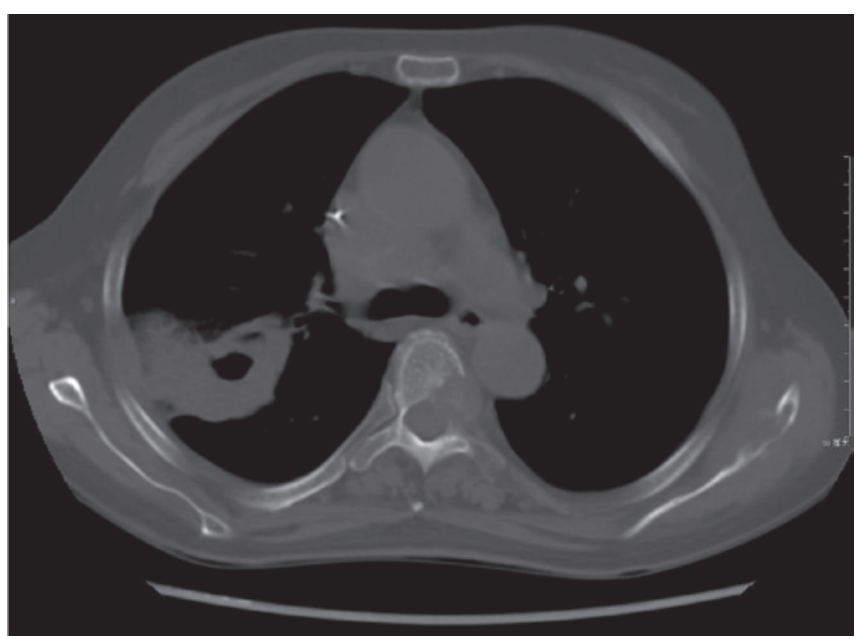

Figure 3. Computed tomography scan of the atlantoaxial joint metastases.

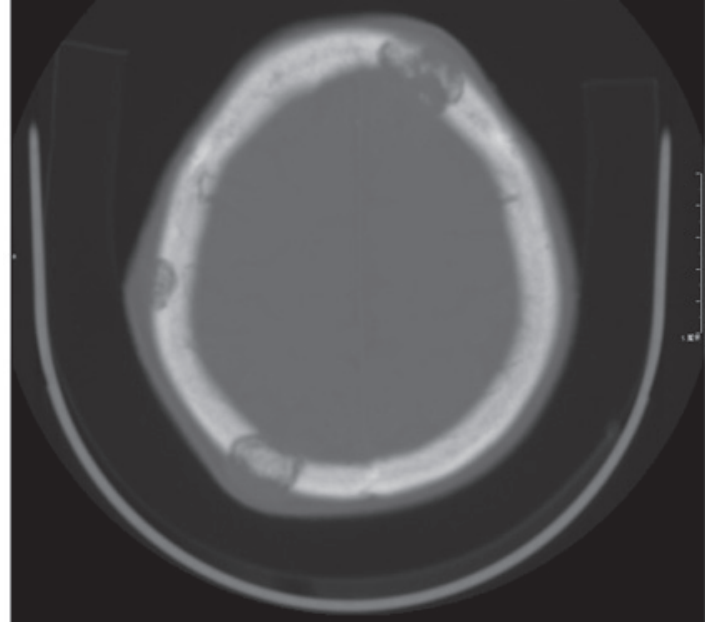

Figure 4. Computed tomography scan of the skull metastases

examination revealed a closed cervix. The uterus was observed to be anteverted and reduced in size, and the bilateral adnexal region was normal. A transvaginal ultrasound demonstrated no abnormalities. A chest CT revealed multiple metastases in the right upper lobe of the lung, scapula and atlantoaxial joint (Figs. 1-3), while a CT of the abdomen revealed no abnormalities. A head CT revealed multiple metastases in the skull, neck and lymph nodes (Fig. 4). Ultrasound examination of masses in the scalp and abdomen revealed destruction of the cranial plate and a hypoechoic mass located in the left flank muscles, respectively. The patient was assigned FIGO stage IV and a WHO score of 13, indicating high risk and poor prognosis (4). Typically, a patient with this diagnosis would be treated with etoposide, methotrexate, actinomycin D, cyclophosphamide and vincristine (EMA-CO) chemotherapy (5), however as the patient was in a poor general condition, alternative systemic chemotherapy, comprising tegafur $(800 \mathrm{mg})$ and actinomycin D $(200 \mu \mathrm{g})$, was administered. During the process of chemotherapy, the patient exhibited itchy skin, a red maculopapular rash, fever and other symptoms. Due to these significant side-effects, systemic chemotherapy was halted on day 4 . Antibiotics ( 0.2 g cefotaxime, every $12 \mathrm{~h}$ ) were administered for 5 days to prevent infection, and the scalp and left flank masses were first injected with methotrexate $(20 \mathrm{mg})$, followed by injection with 5 -fluorouracil (250 mg). During chemotherapy, $\beta$-HGG levels decreased from $3,171 \mathrm{IU} / 1$ to $1,763 \mathrm{IU} / 1$. Following remission of the side-effects, the patient completed the last course for four days. However, at the conclusion of the courses of systemic and local chemotherapy, $\beta$-HCG levels had increased to 3,704 IU/1. Laboratory tests indicated that hemoglobin levels, platelet numbers and C-reactive protein levels were $59 \mathrm{~g} / \mathrm{l}$, $59 \times 10^{9} / 1$ and $86.4 \mathrm{~g} / \mathrm{l}$, respectively. The patient and their family elected to end treatment. Subsequently, the patient succumbed to infection six months after initial admission, due to tumor consumption and organ insufficiency.

\section{Discussion}

Choriocarcinoma is a highly malignant tumor that is classified among the gestational trophoblastic diseases (1). 
Choriocarcinoma is typically present within a hydatiform mole, and primarily occurs during the fertile period. Choriocarcinoma incidence is rare following menopause (1). Trophoblastic cells possess an affinity for blood vessels; therefore trophoblastic tumors exhibit a tendency to metastasize via the hematogenous route (6). In the present report, a particularly rare case of neoplasm, which developed 20 years subsequently to the onset of menopause and 42 years following the patient's final pregnancy, was outlined.

Choriocarcinomas are aggressive malignancies, which may be separated into two groups: Non-gestational choriocarcinoma (NGCO), which typically arises from the gonadal organs, but may also occur in extragonadal primary sites; and gestational choriocarcinoma (GCO), which is derived from any form of previously normal or abnormal pregnancy, such as a hydatidiform mole, spontaneous abortion or ectopic pregnancy (7). Due to its poorer prognosis, NGCO requires more aggressive therapy with numerous chemotherapeutic agents (8), and may also require surgical treatment. DNA analysis is a reliable method for distinguishing between the two categories (8). Exman et al (9) hypothesized that polymorphic analysis of tumor DNA was necessary for diagnosis. Rare cases of NGCO have also been reported at multiple locations, including the ovaries (9), lungs (10), urinary bladder (11), stomach (12) and vulva (13). The current patient presented with extra-uterine choriocarcinoma, and the primary symptoms exhibited were pulmonary lesions, bone metastases and skin masses. The patient was not eligible for surgical treatment of the tumors due to poor general condition, so it could not be confirmed whether the patient exhibited NGCO or GCO with multiple lung, skull, neck, lymph node and skin metastases. The most common sites for choriocarcinoma metastases are the lungs and vulvovaginal region, followed by the brain and liver (14). Alternative sites of metastasis, including the skin, gastrointestinal tract, kidney, breast and bone, are rarely observed (15).

A total of $75 \%$ of FIGO stage IV choriocarcinoma patients are expected to achieve complete or prolonged remission when treated with multiagent chemotherapy regimens, including MAC (methotrexate, actinomycin-D and cyclophosphamide) and CHAMOCA (cyclophosphamide, hydroxycarbamide, doxorubicin, actinomycin D, methotrexate, melphalan and vincristine) (16). Currently, the most commonly used alternatives to the aforementioned multiagent chemotherapy regimens are EMA-CO or fluorouracil-based chemotherapy regimens. Due to the poor condition of the current patient, systemic chemotherapy with tegafur and actinomycin D was administered, and the scalp and left flank masses were directly injected with methotrexate and 5-fluorouracil. The patient and their family subsequently elected to end treatment, and the patient succumbed to the disease.

In conclusion, the current case presented unusual findings with regard to choriocarcinoma, and thus, differentiating between primary tumors of the lungs or other organs and metastases, and choriocarcinoma was difficult. This study indicates that in cases where one isolated lesion in the lung, bone or other organ is identified in a postmenopausal female, the possibility of choriocarcinoma must be considered. Subsequently, combination chemotherapy may be administered with curative intent or with the aim of prolonging life or to palliate symptoms.However, in certain cases conservative NGCO therapy may be insufficient and thus, surgical excision may be required.

\section{References}

1. Morrow CP, Kletzky OA, Disaia PJ, Townsend DE, Mishell DR and Nakamura RM: Clinical and laboratory correlates of molar pregnancy and trophoblastic disease. Am J Obstet Gynecol 128: 424-430, 1977.

2. Lee JH, Park CW, Chung DH and Kim WK: A case of lumbar metastasis of choriocarcinoma masquerading as an extraosseous extension of vertebral hemangioma. J Korean Neurosurg Soc 47: $143-147,2010$.

3. Cole LA, Khanlian SA, Muller CY, Giddings A, Kohorn E and Berkowitz R: Gestational trophoblastic disease: 3. Human chorionic gonadotrophin-free beta-subunit, reliable marker of placental site trophoblastic tumors. Gynecol Oncol 102: 160-164, 2006.

4. Sierra-Bergua B, Sánchez-Marteles M, Cabrerizo-García JL and Sanjoaquin-Conde I: Choriocarcinoma with pulmonary and cerebral metastases. Singapore Med J 49: e286-e288, 2008.

5. El-Helw LM and Hancock BW: Treatment of metastatic gestational trophoblastic neoplasia. Lancet Oncol 8: 715-724, 2007.

6. Morgan JM and Lurain JR: Gestational trophoblastic neoplasia: An update. Curr Oncol Rep 10: 497-504, 2008.

7. Guo J, Zhong C, Liu Q, Xu J, Zheng Y, Xu S, Gao Y, Guo Y, Wang Y, Luo Q and Jiang J: Intracranial choriocarcinoma occurrence in males: Two cases and a review of the literature. Oncol Lett 6: 1329-1332, 2013.

8. Jacobs AJ, Newland JR and Green RK: Pure choriocarcinoma of the ovary. Obstet Gynecol Surv 37: 603-609, 1982.

9. Exman P, Takahashi TK, Gattás GF, Cantagalli VD, Anton C, Nalesso F and Diz Mdel P: Primary ovary choriocarcinoma: Individual DNA polymorphic analysis as a strategy to confirm diagnosis and treatment. Rare Tumors 5: 89-92, 2013.

10. Di Crescenzo V, Laperuta P, Napolitano F, Carlomagno C, Garzi A and Vitale M: An unusual case of primary choriocarcinoma of the lung. BMC Surg 13 (Suppl 2): S33, 2013.

11. Gallagher L, Lind R and Oyasu R: Primary choriocarcinoma of the urinary bladder in association with undifferentiated carcinoma. Hum Pathol 15: 793-795, 1984.

12. Matsunaga N, Hayashi K, Futagawa S, Fukuda T, Takahara O, Yoshida K and Maeda H: Primary choriocarcinoma of the stomach presenting as gastrointestinal hemorrhage: Report of a case. Radiat Med 7: 220-222, 1989.

13. Weiss S, Amit A, Schwartz MR and Kaplan AL: Primary choriocarcinoma of the vulva. Int J Gynecol Cancer 11: 251-254, 2001.

14. Milenković V, Lazović B, Mačvanski M, Jeremić K and Hrgović Z: Clinical outcome of a FIGO stage IV gestational choriocarcinoma. Case Rep Oncol 6: 504-507, 2013.

15. Singh S, Sardhana M, Sharma S and Chitralkar P: Choriocarcinoma presenting as an isolated bone marrow metastasis - a case report. Ecancermedicalscience 8: 393, 2014.

16. FIGO Committee on Gynecologic Oncology: Current FIGO staging for cancer of the vagina, fallopian tube, ovary, and gestational trophoblastic neoplasia. Int J Gynaecol Obstet 105: 3-4, 2009. 